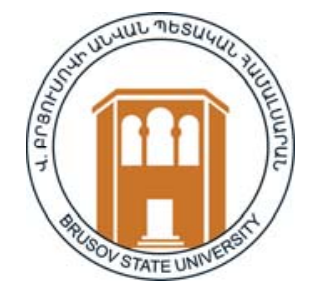

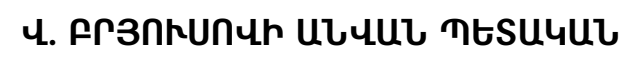
CUUULUURUL

ГОСУДАРСТВЕННЫЙ УНИВЕРСИТЕТ

ИМЕНИ В. БРЮСОВА

BRUSOV STATE UNIVERSITY

คulคtr

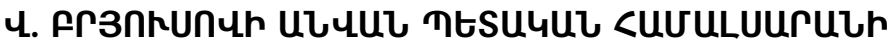
ВЕСТНИК ГОСУДАРСТВЕННОГО УНИВЕРСИТЕТА ИМЕНИ

B. БРЮСОВА

BULLETIN OF BRUSOV STATE UNIVERSITY

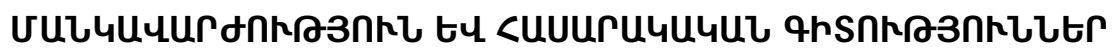

ПЕДАГОГИКА И СОЦИАЛЬНЫЕ НАУКИ

PEDAGOGY AND SOCIAL SCIENCES

2(55)

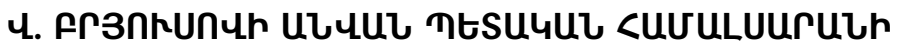

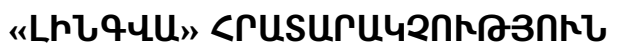

เГヒบuし - 2021 
<S? 37.037.1

DOI: $10.51307 / 182931072015233260 / 21.2-14$

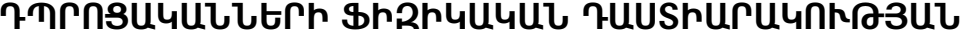

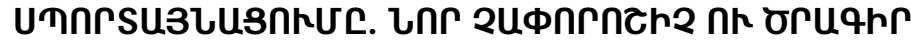

\section{tกนtUS uบuひtUกч}

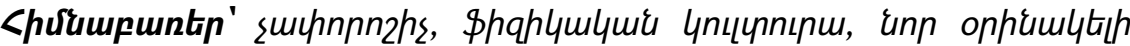

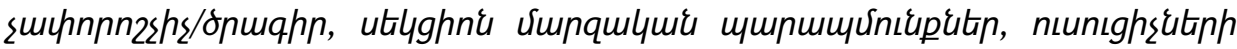
шquчиuluikmgnuर

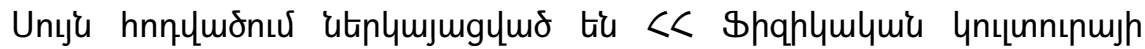

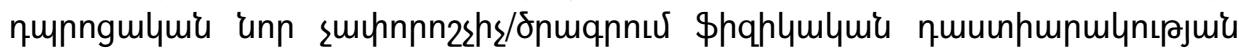

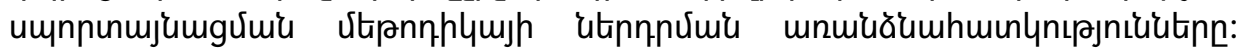

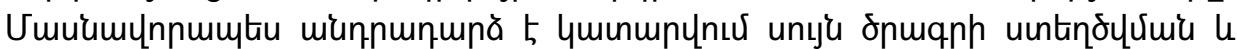

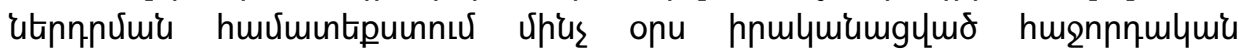

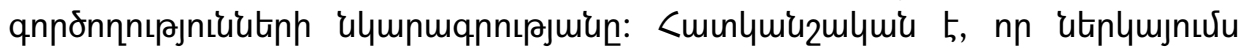

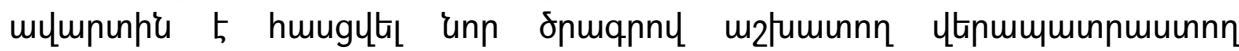

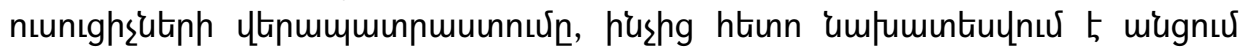

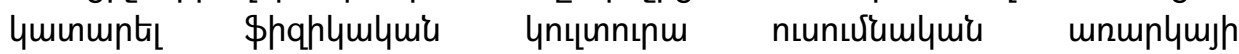

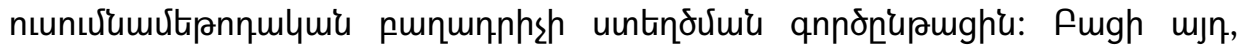

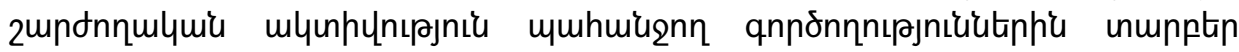

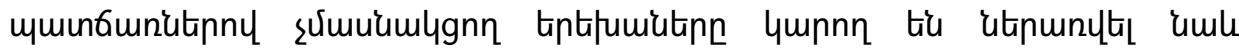

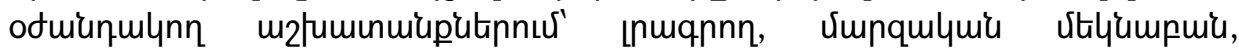

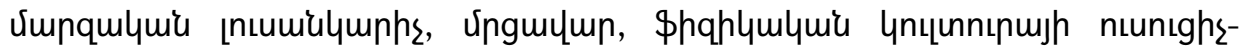

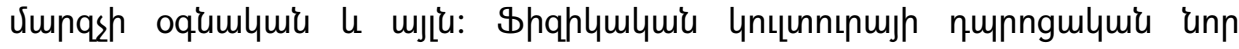

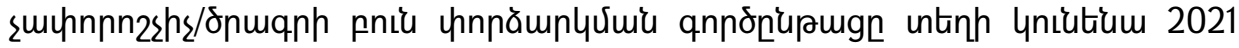
plulyminh utumuntuiptiphg:

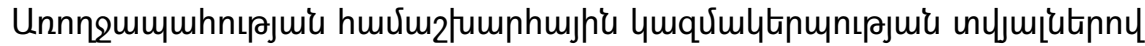

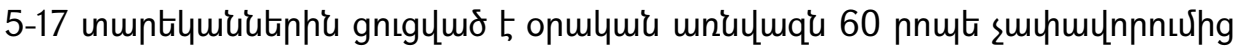

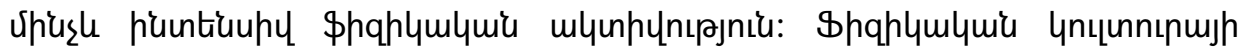

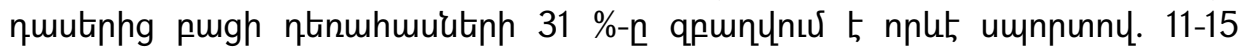

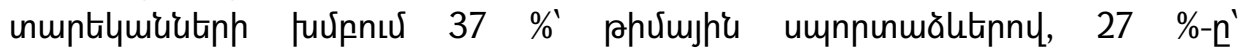

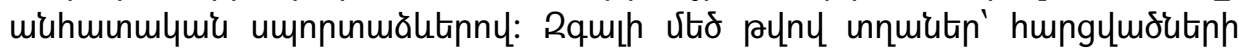

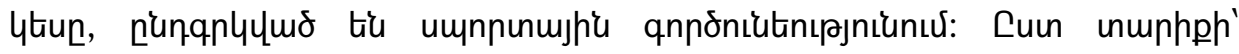

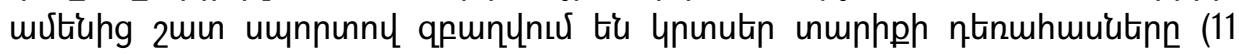

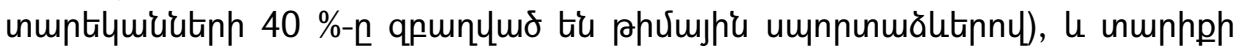

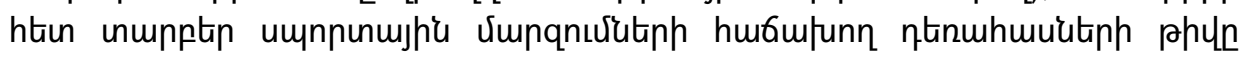




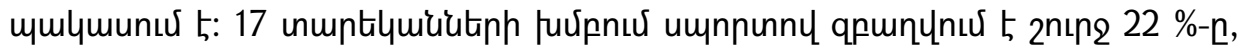

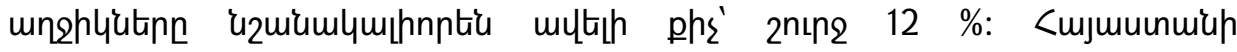

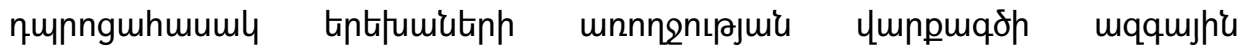

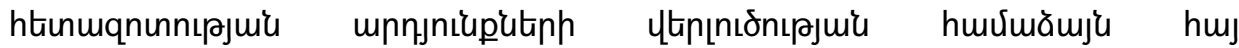

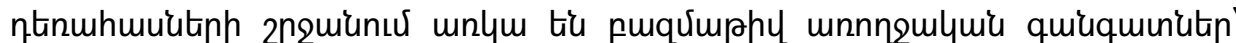

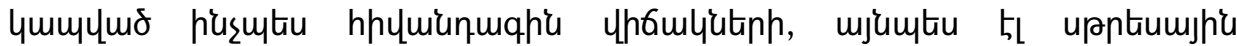

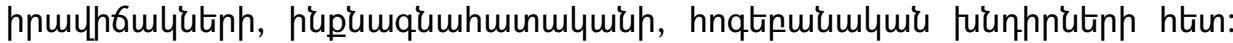

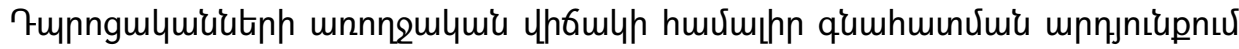

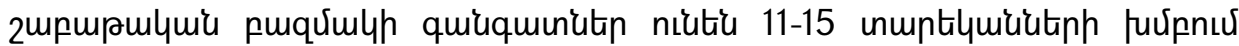

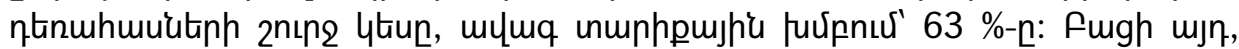

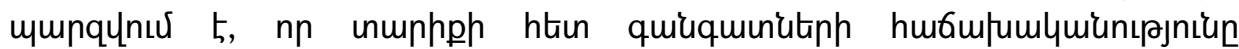

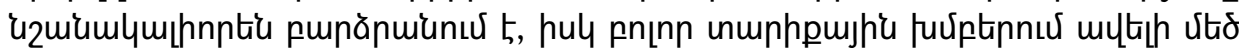

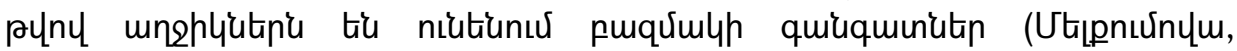

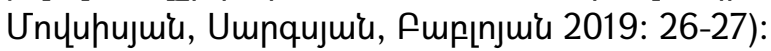

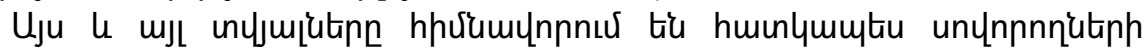

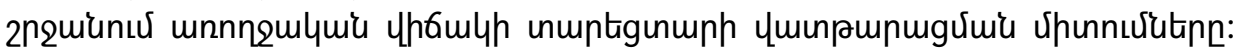

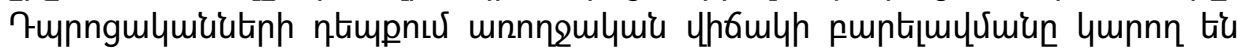

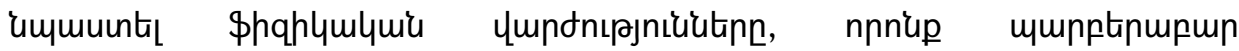

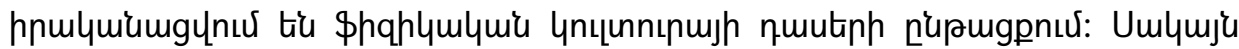

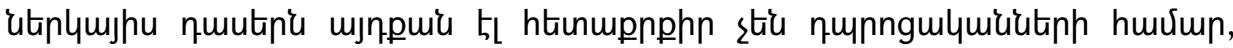

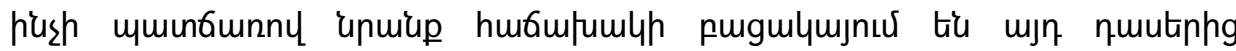

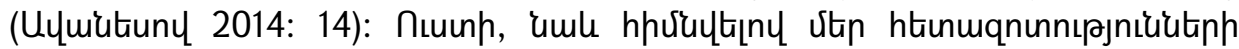

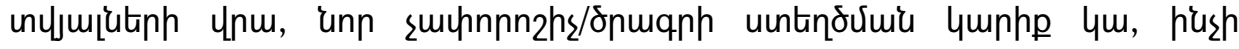

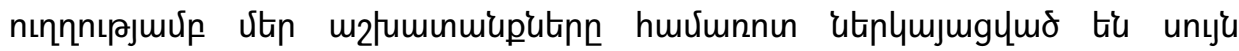

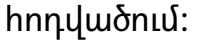

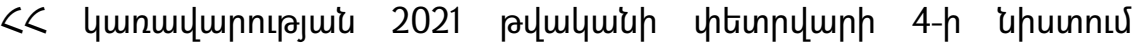

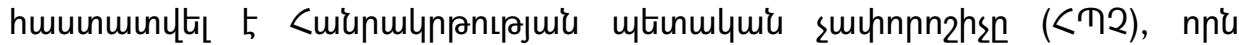

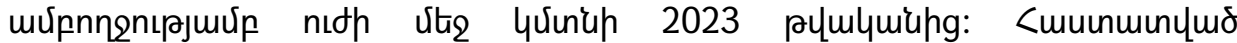

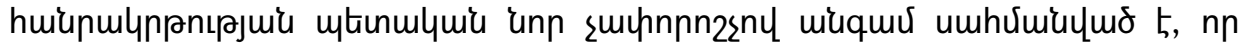

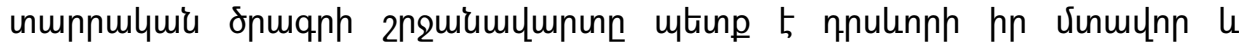

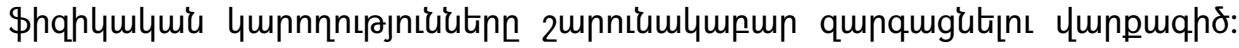

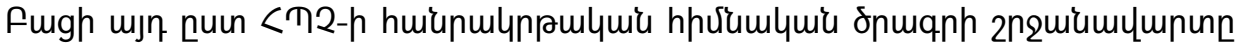

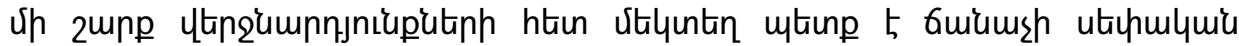

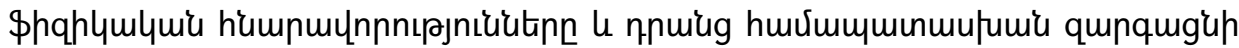

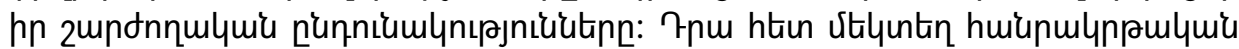

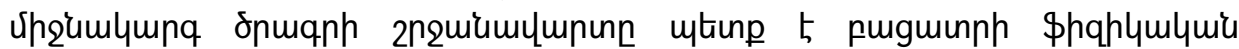

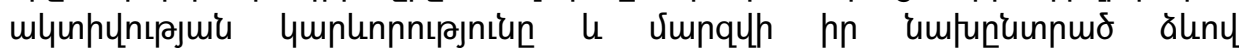

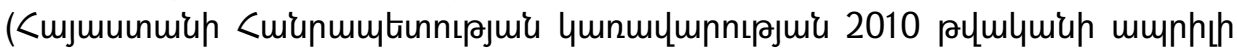

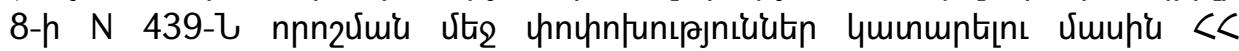

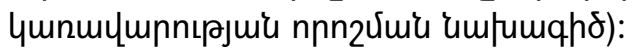




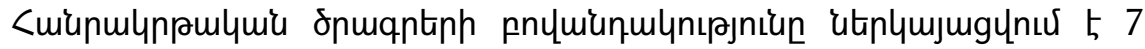

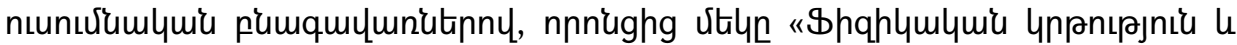

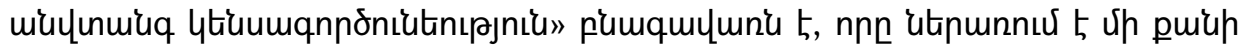

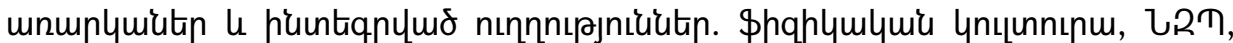

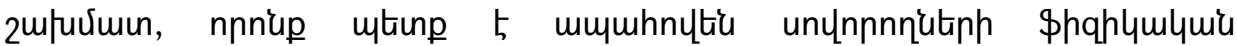

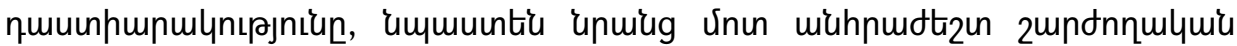

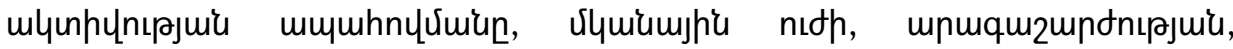

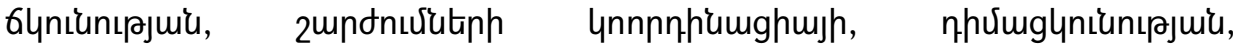

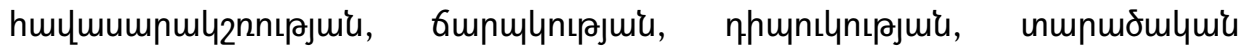

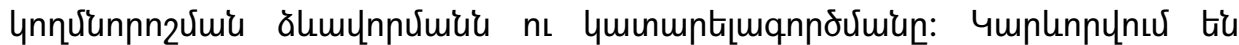

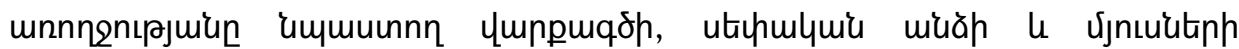

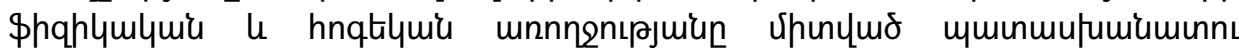

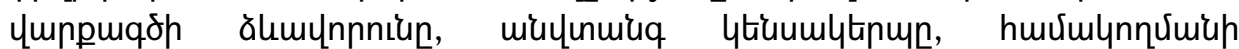

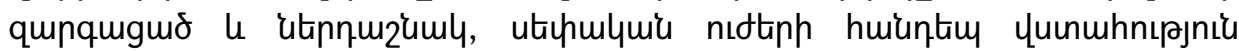

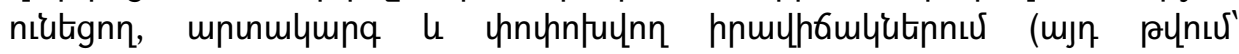

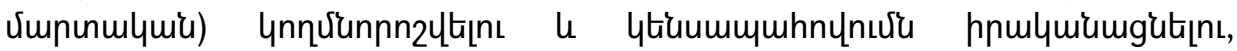

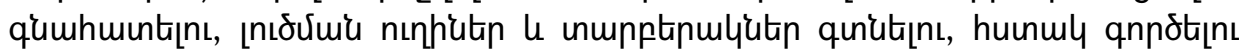

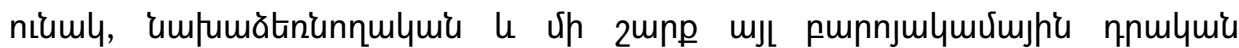

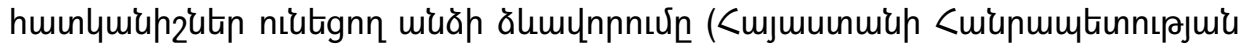

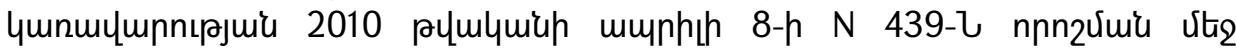

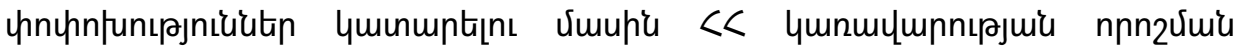
umpumqh):

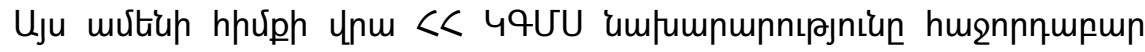

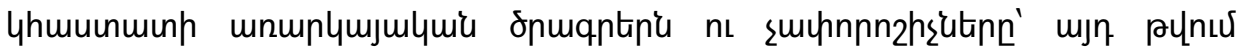

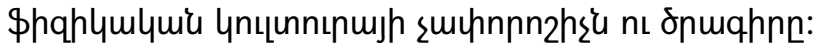

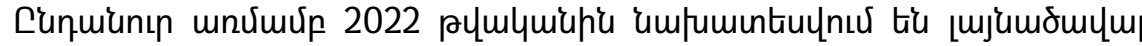

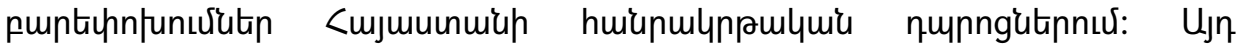

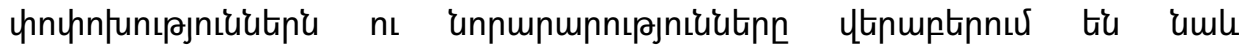

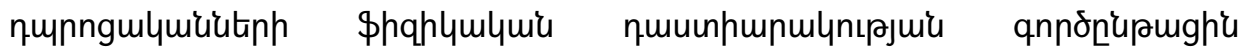

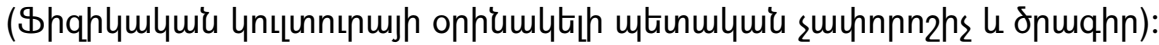

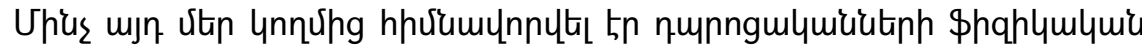

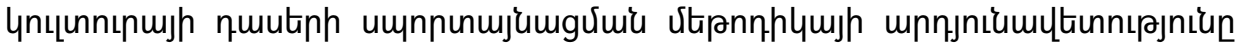
(Uyuituruny 2014: 24):

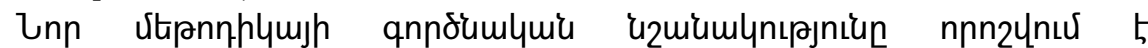

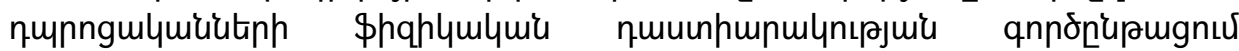

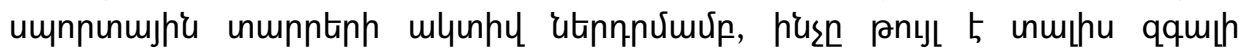

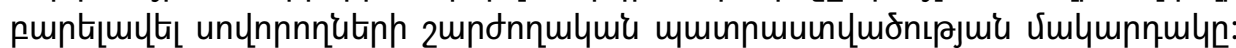

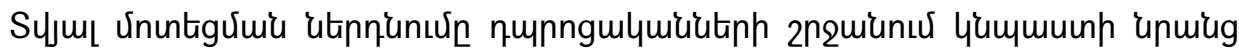
2undnnulymi nün

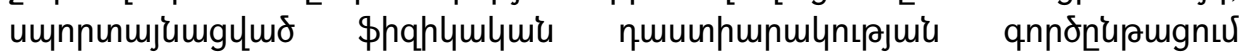




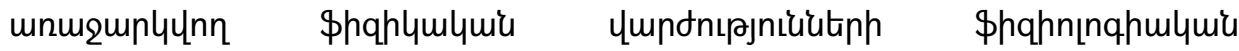

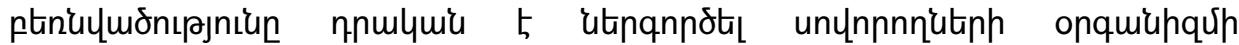

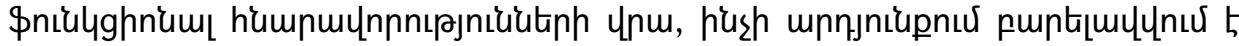
cumle unuing unnngulymis yh6ulyn:

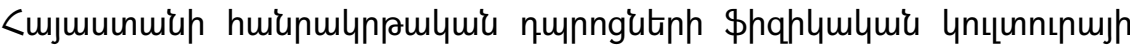

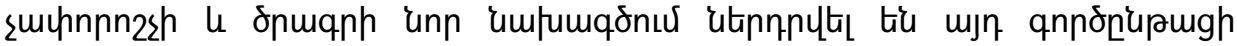
umnnunujumgưuiu unmpntip:

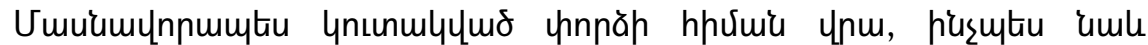

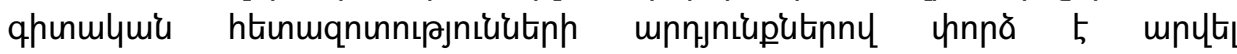

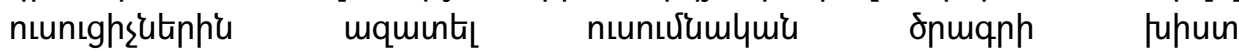

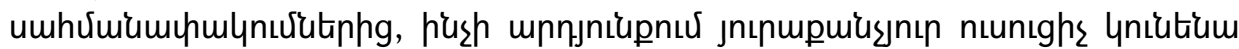

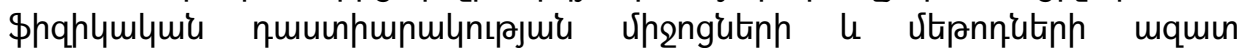

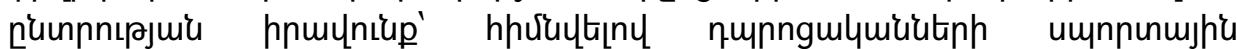

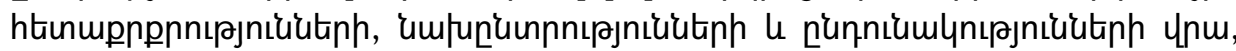

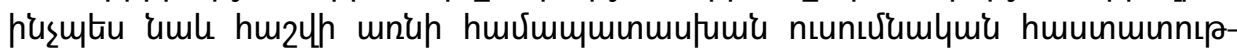

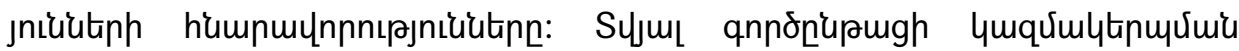

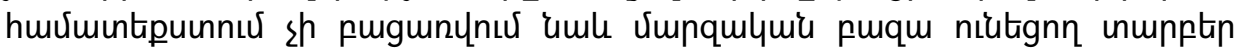

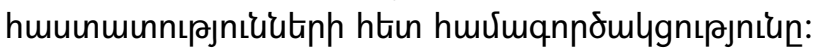

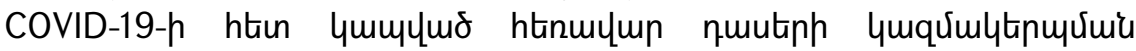

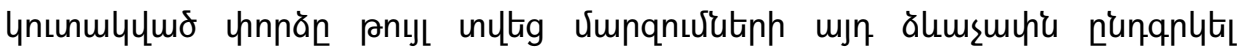

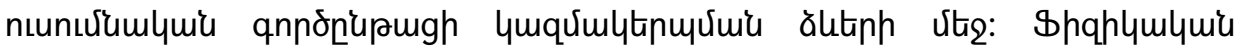

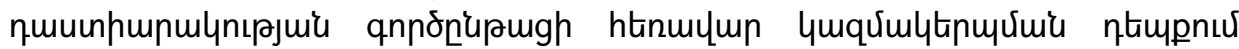

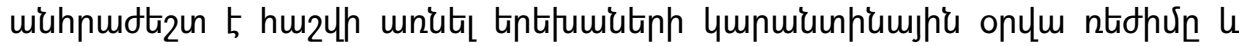

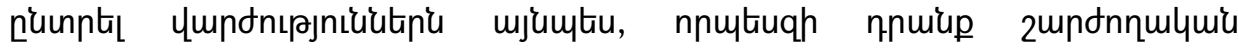

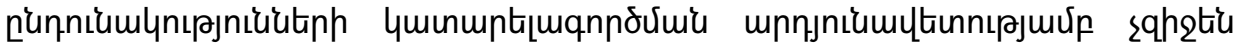

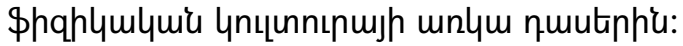

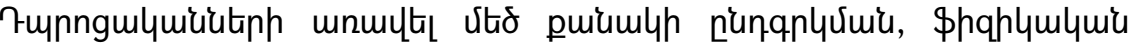

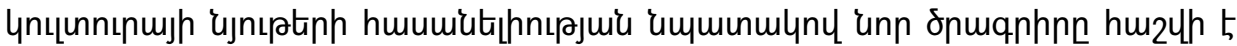

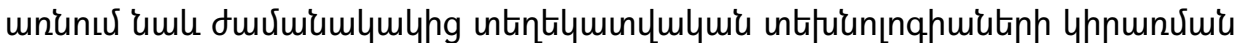

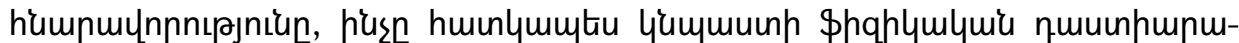

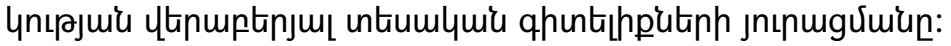

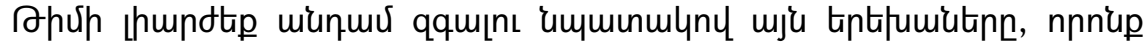

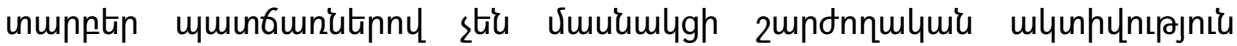

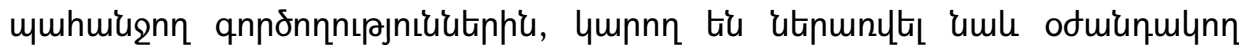

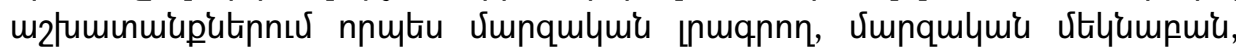

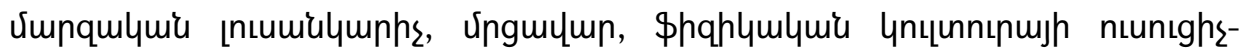
vimnqsh oquiulyuir u mjı:

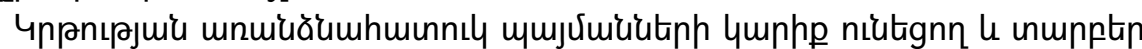

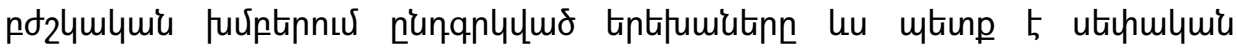

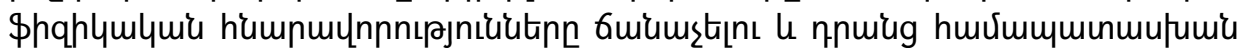




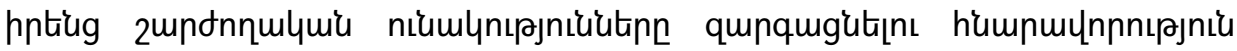
niutiumis:

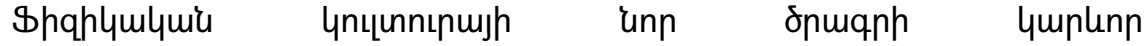

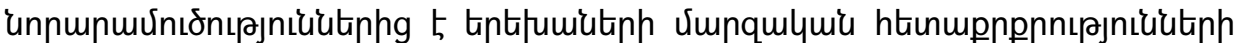

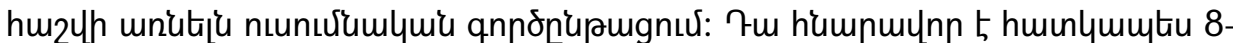

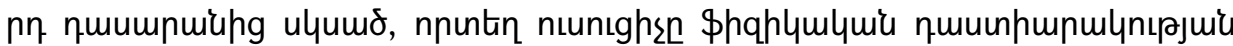

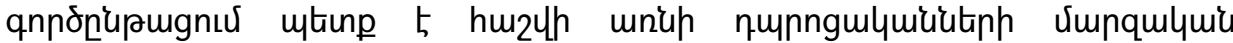

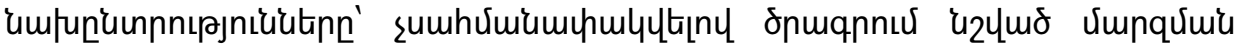

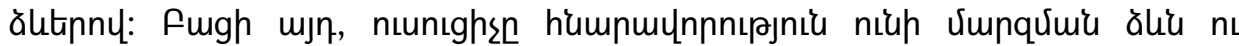

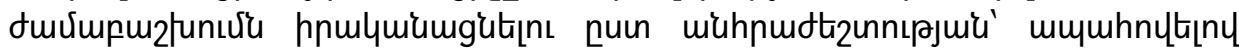

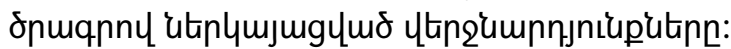

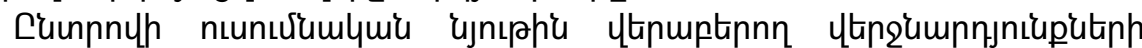
mumhny

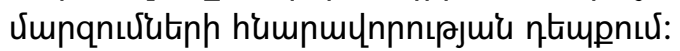

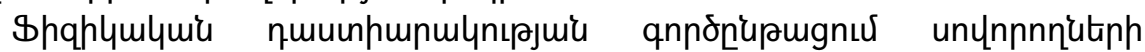

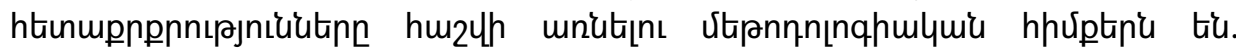

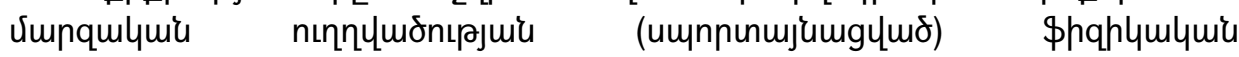

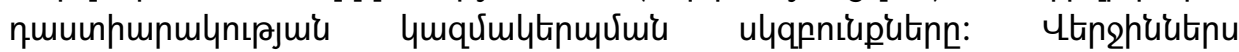

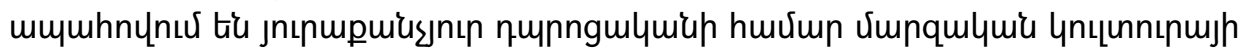

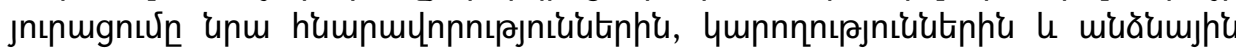

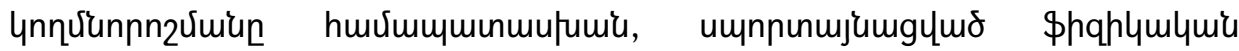

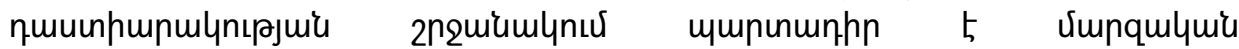

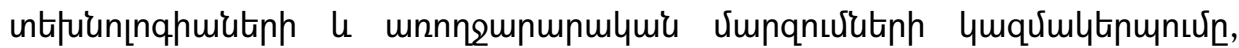

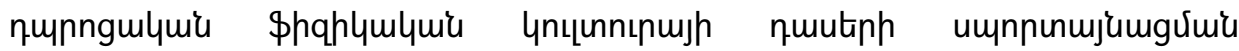

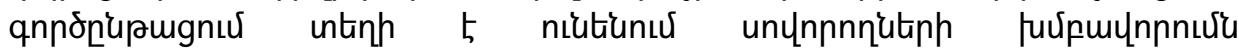

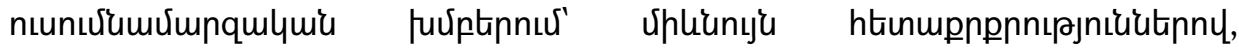

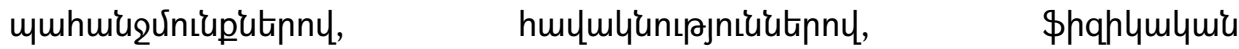

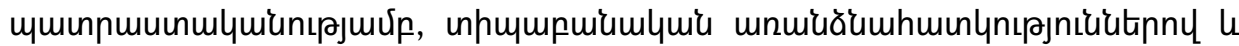

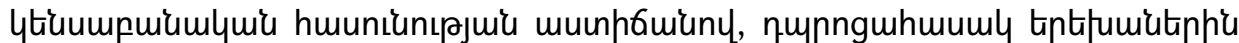

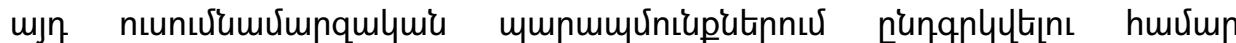

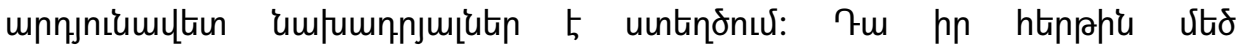

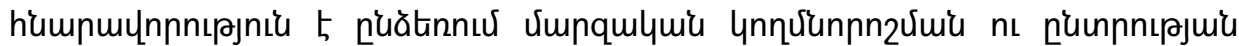

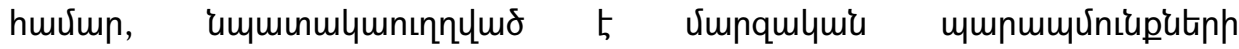

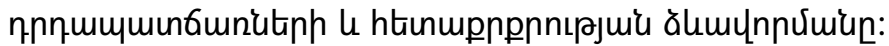

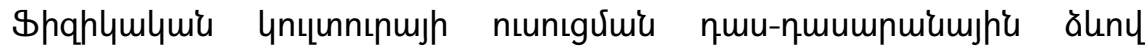

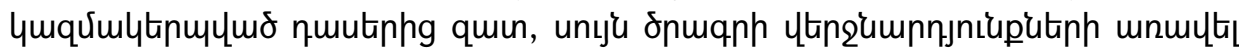

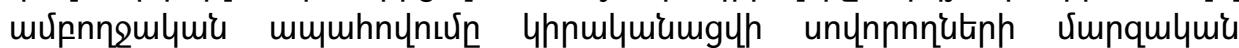

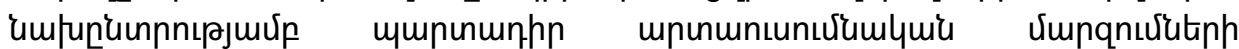
huraufutiny: 


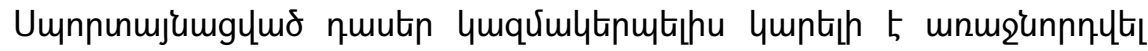

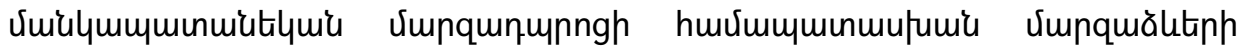

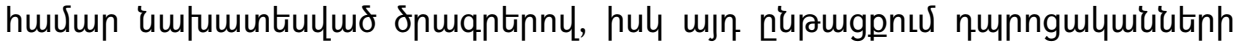

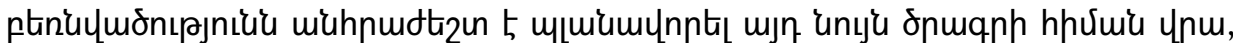

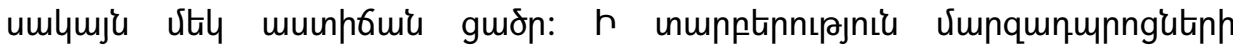

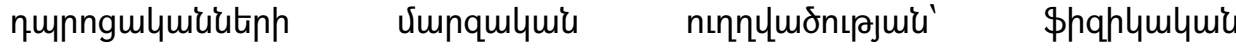

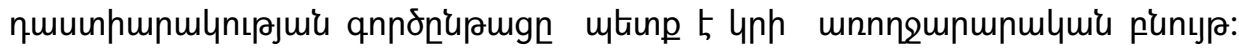

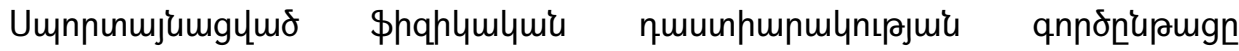

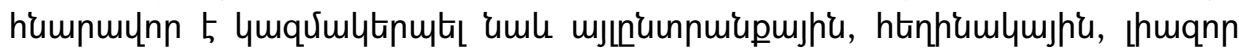

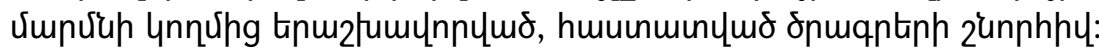

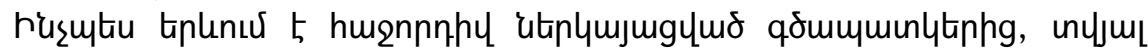

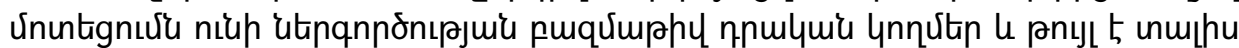

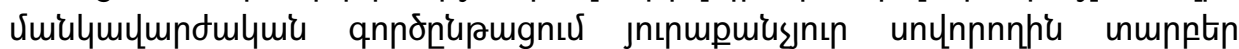

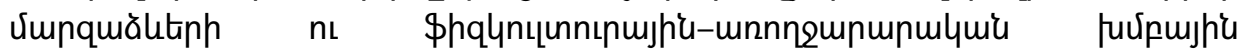

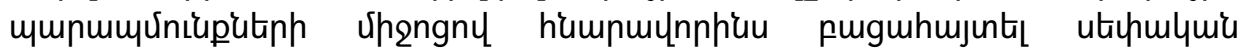

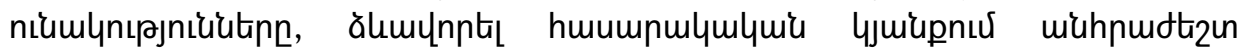

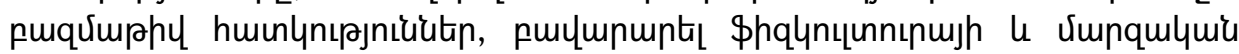

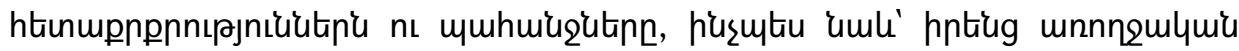
पh6urle (untiu' qommuinltip 1):

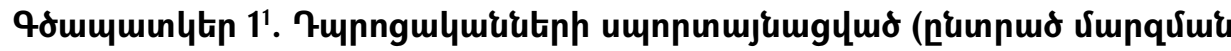

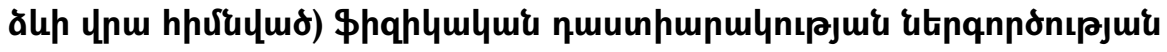

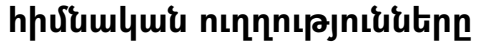

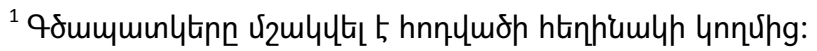




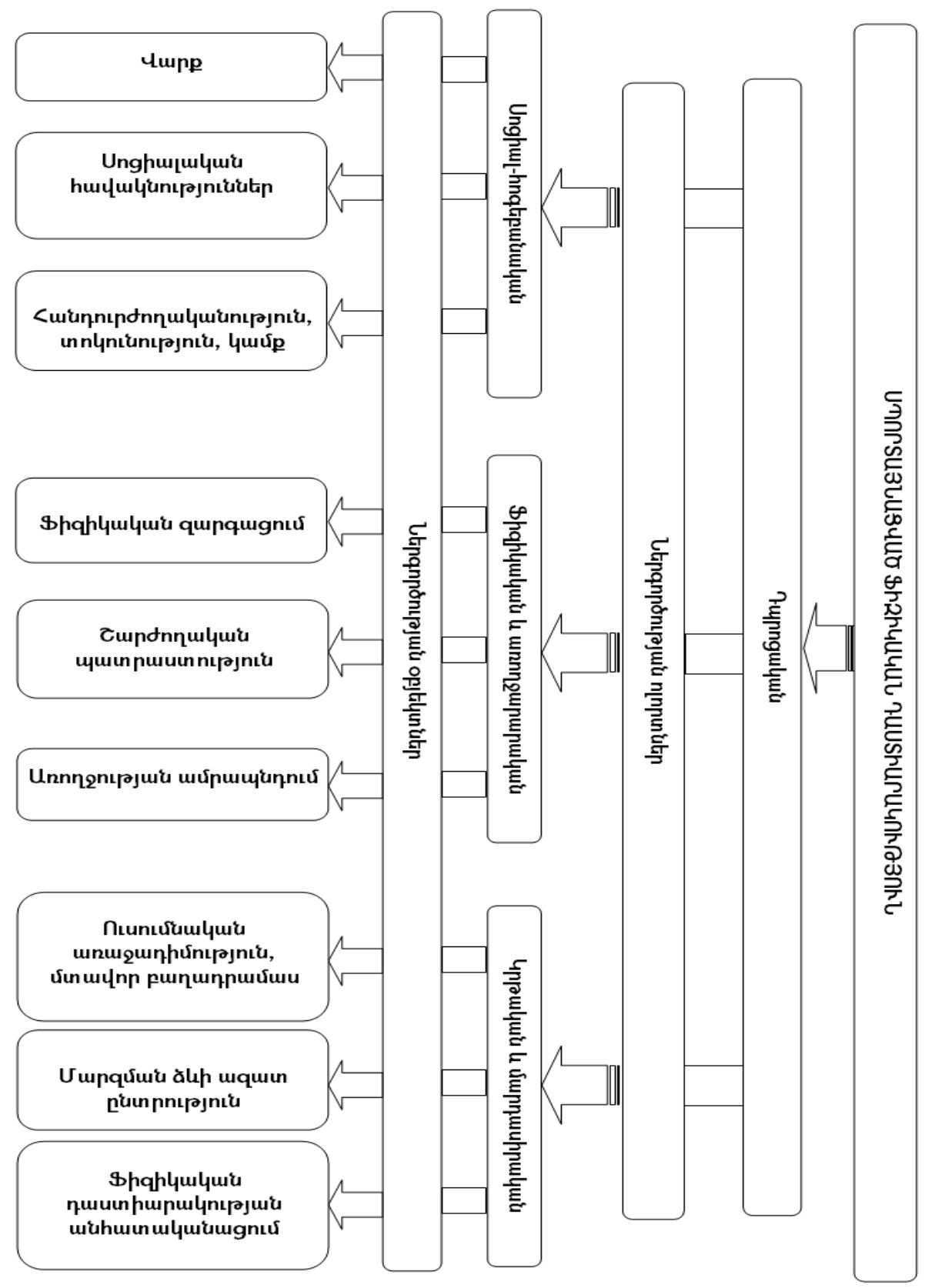

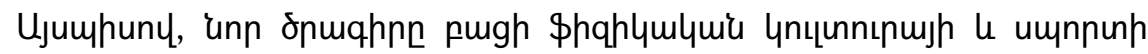

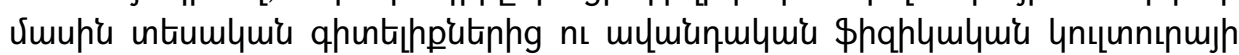

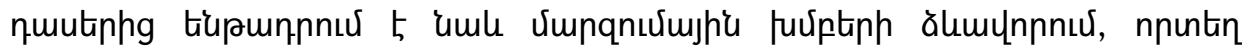




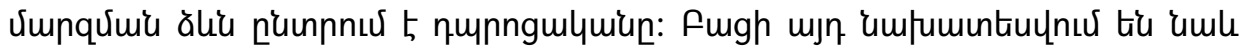

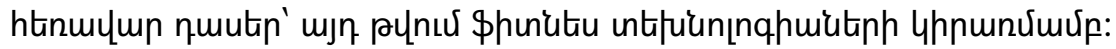

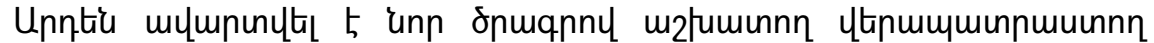

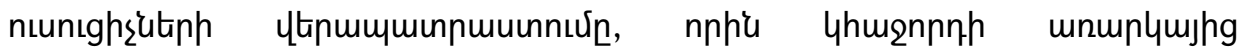

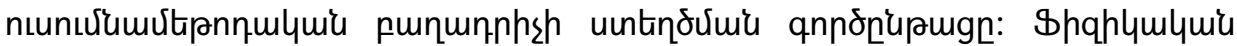

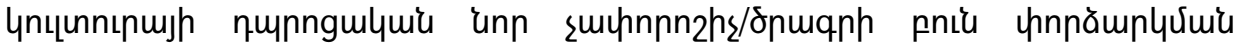

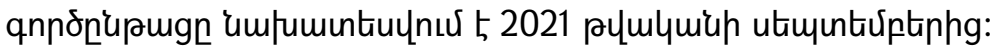

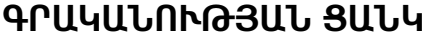

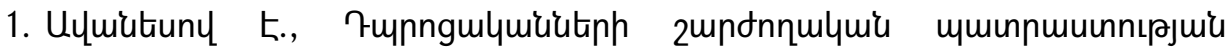

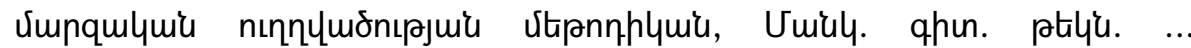

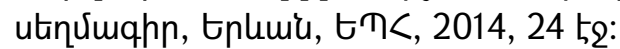

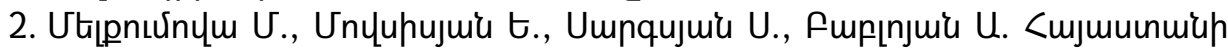

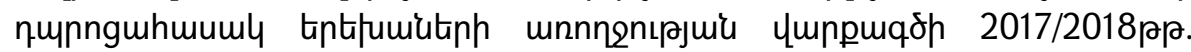

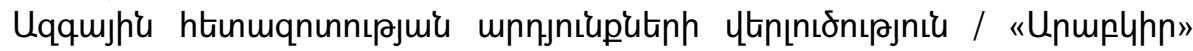

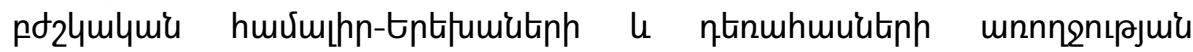
huuunhunnum, 2019, 42 52:

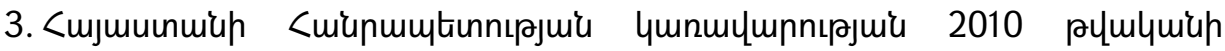

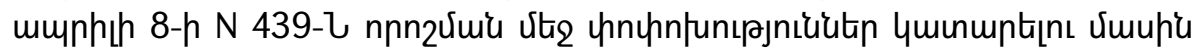

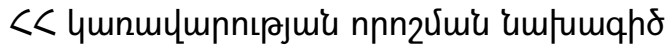

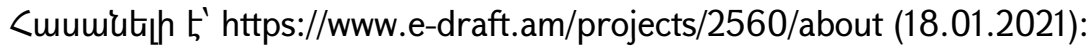

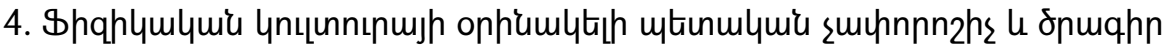
cuumut_h h' $^{\prime}$ http://escs.am/am/news/6711 (22.01.2021):

\section{REFERENCES}

1. Avanesov E'. Dprocakanneri sharjhoghakan patrastowt'yan marzakan owghghvac'owt'yan met'odikan: Mank. git. t'ekn. ... seghmagir, Er\&an: EPH, 2014.-24 e'j:

2. Melqowmova M., Movsisyan E., Sargsyan S., Babloyan A. Hayastani dprocahasak erexaneri ar'oghjowt'yan varqagc'i 2017/2018t't'. Azgayin hetazotowt'yan ardyownqneri verlowc'owt'yown / "Arabkir» bjhshkakan hamalir-Erexaneri \& der'ahasneri ar'oghjowt'yan institowt, 2019, 42 e'j:

3. Hayastani Hanrapetowt'yan kar'avarowt'yan 2010 t'vakani aprili 8-i N 439-N oroshman mej p'op'oxowt'yownner katarelow masin HH kar'avarowt'yan oroshman naxagic'

Hasaneli e" https://www.e-draft.am/projects/2560/about (18.01.2021):

4. Fizikakan kowltowrayi o'rinakeli petakan chap'oroshich \& c'ragir Hasaneli e" http://escs.am/am/news/6711 (22.01.2021): 


\section{ЭРНЕСТ АВАНЕСОВ - СПОРТИЗАЦИЯ ФИЗИЧЕСКОГО ВОСПИТАНИЯ ШКОЛЬНИКОВ: НОВЫЙ СТАНДАРТ И ПРОГРАММА}

Ключевые слова: государственный стандарт, примерная программа по фризической культуре, секционные спортивные занятия, либерализация преподавателей

В статье представлены особенности внедрения методики спортизированного ффизического воспитания В новый стандарт по фризической культуре школ РА. В частности рассматриваются последовательные действия, которые осуществлялись до сегодняшнего дня в контексте создания и внедрения данной программы. Сама программа будет апробирована с сентября 2021 года.

\section{ERNEST AVANESOV - SPORTIFICATION OF PHYSICAL EDUCATION OF SCHOOLCHILDREN: NEW STANDARD AND PROGRAM}

Keywords: state standard, model curriculum of physical education, sectional sport trainings, liberalization of teachers

The article presents the peculiarities of introducing the methodology of sportification of Physical Education into the new school curriculum/program of Physical Education, RA. In particular, reference is made to the description of successive actions taken to date, within the context of developing and implementing this program. The testing of the program is scheduled for September 2021. 